Title:

\title{
Giant Magnetoresistance Materials for Magnetic Recording Technology
}

\section{Authors:}

Submitted to:

R. H. Heffner, MST-10

C. D. Adams, MST-7

E. L. Brosha, MST-11

J. Brug, Hewlett Packard

S. D. Conradson, MST-11

F. H. Garzon, MST-11

M. Hawley, MST-CMS

M. F. Hundley, MST-10

Q. Jia, MST-STC

J. Nickel, Hewlett Packard

M. B. Salomon, U. Illinois

T. A. Tyson, MST-11

X. Di Wu, MST-STC

DOE Office of Scientific and Technical

Information (OSTI)

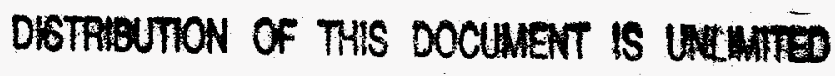
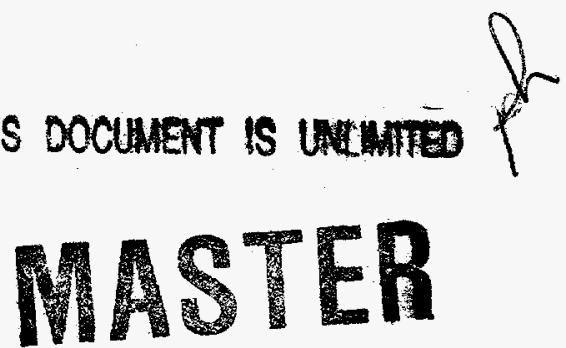

\section{Los Alamos}

NATIONAL L ABORATORY

Los Alamos National Laboratory, an affirmative action/equal opportunity employer, is operaled by the University of Califomia for the U.S. Department of Energy under contract W-7405-ENG-36. By acceptance of this article, the publisher recognizes that the U.S. Govemment retains a nonexclusive, royaltyfree license 10 publish or reproduce the published form of this contribution, or to allow others to do so, for U.S. Government purposes. Los Alamos Nalional Laboralory requests that the publisher identify this article as work pertormed under the auspices of the U.S. Department of Energy. Los Alamos National Laboratory strongly supports academic freedom and a researcher's right to publish; as an institution, however, the Laboratory does not endorse the vieupoint of a publication or guarantee its technical correctness. 


\section{DISCLAMRER}

Partions of this doement migy be illegible in electronic image produets. Inoges are produced from the best available original doenoneret 


\section{DISCLAIMER}

This report was prepared as an account of work sponsored by an agency of the United States Government. Neither the United States Government nor any agency thereof, nor any of their employees, makes any warranty, express or implied, or assumes any legal liability or responsibility for the accuracy, completeness, or usefulness of any information, apparatus, product, or process disclosed, or represents that its use would not infringe privately owned rights. Reference herein to any specific commercial product, process, or service by trade name, trademark, manufacturer, or otherwise does not necessarily constitute or imply its endorsement, recommendation, or favoring by the United States Government or any agency thereof. The views and opinions of authors expressed herein do not necessarily state or reflect those of the United States Government or any agency thereof. 


\title{
Giant Magnetoresistance Materials for Magnetic Recording Technology
}

\author{
Robert H. Heffner, Chris D. Adams, Eric L. Brosha, Steven D. Conradson, \\ Fernando H. Garzon, Marilyn Hawley, Michael F. Hundley, \\ Quanxi Jia, Trevor A. Tyson, and Xin Di Wu \\ Los Alamos National Laboratory \\ Jim Brug and Janice Nickel \\ Hewlett Packard \\ Myron B. Salomon \\ University of Illinois
}

\begin{abstract}
This is the final report of a two-year, Laboratory Directed Research and Development (LDRD) project at Los Alamos National Labortory (LANL). This work focused on a class of transition-metal-oxide (TMO) materials $\left(\mathrm{LaMnO}_{3}\right.$ doped with $\mathrm{Ca}, \mathrm{Ba}$, or $\left.\mathrm{Sr}\right)$ that exhibits an insulator-to-metal transition near a ferromagnetic phase transition temperature. This yields a very large magnetoresistance; thus these materials may have important uses as magnetic sensors in a variety of applications, ranging from automobiles to read heads for magnetic storage. In addition, the transport current in the ferromagnetic state is likely to be very highly polarized, which means that additional device applications using the phenomena of spin-polarized tunneling can be envisioned. Use of these materials as magnetic sensors depends upon learning to control the synthesis parameters (principally temperature, pressure and composition) to achieve a specific carrier concentration and/or mobility. A second challenge is the high magnetic fields ( $\geq 1$ Tesla) currently required to achieve a large change in resistance. We began an investigation of two novel approaches to this field-sensitivity problem, involving the development of multilayer structures of the TMO materials. Finally, we began to explore the use of epitaxial strain as a means of changing the transport properties in thin-film multilayers.
\end{abstract}

\section{Background and Research Objectives}

This work focused on a class of transition-metal-oxide (TMO) materials - $\mathrm{LaMnO}_{3}$ doped with $\mathrm{Ca}, \mathrm{Ba}$, or $\mathrm{Sr}$ - that exhibits an insulator-to-metal transition near its ferromagnetic phase transition temperature $T_{c}[1]$. This yields a very large magnetoresistive (MR) behavior: a change in resistance $R$ with applied magnetic field $(\Delta R / R \cong-90 \%)$ near $T_{C}$. These materials may have important uses as magnetic sensors in a

\footnotetext{
- Principal Investigator, email: heffner@rayleigh.lanl.gov
} 
variety of applications, ranging from automobiles to read heads for magnetic storage. In addition, the transport current in the ferromagnetic state is likely to be very highly polarized, which means that additional device applications using the phenomena of spinpolarized tunneling can be envisioned. Advances in storage media, heads and drives is driven by a continual need to increase the information storage density in rewritable data storage systems. The consensus candidates to achieve a storage-density goal of $1 \mathrm{~GB} / \mathrm{in}^{2}$ are various types of MR sensors. The oxide materials investigated by our team are of potential interest for the next generation of read heads, in order to reach recording densities exceeding $10 \mathrm{~GB} / \mathrm{in}^{2}$.

Use of these materials as magnetic sensors or devices of any kind entails overcoming several fundamental challenges. First and foremost is learning to control the synthesis parameters (principally temperature, pressure and composition) to achieve a specific carrier concentration and/or mobility. This is a major challenge for all TMO film development. As discussed in detail below, we believe we have made significant progress in this area. A second challenge for the application to magnetic read heads is the high magnetic fields ( $\geq 1$ Tesla) currently required to achieve a large change in resistance. Applications in magnetic recording require fields $<100$ Oe. We conceived of two novel approaches to this field-sensitivity problem, both of which involve developing multilayer structures of the TMO materials. Finally, we hegan to explore various ways to exploit the extreme sensitivity of the conductivity to pressure found in bulk samples; these ideas involve the use of epitaxial strain as a means of changing the transport properties in thinfilm multilayers.

\section{Importance to LANL's Science and Technology Base and National R\&D} Needs

\section{Science and Technology Base}

The subject of Advanced Materials has been identified as a Laboratory core competency and increasing emphasis is now placed on developing materials related to improved economic competitiveness. Work in partnership with industry and fundamental science of high quality remain two of the Laboratory's important goals. The project described here on MR materials meets all of these criteria. In addition, the synthesis and characterization of these materials strengthens the capabilities in the the Materials Science and Technology (MST) Division, as well as the Los Alamos Neutron Science Center (LANSCE), an important national user facility. 


\section{Program Development Efforts}

The future use of TMO materials in device applications is becoming widely appreciated due to the extraordinary variety of phenomena that can be exploited. These include high temperature superconductivity, MR materials, ferroelectrics, etc. In addition to applications of MR materials in magnetic recording, for example, one can hopefully exploit the polarized nature of the transport current in these materials, and develop a switching device that is sensitive to external fields. Our research was focused on learning how to manifest these possibilities.

Our program development efforts initially targeted the magnetic recording industry, through a consortium of companies (HP, IBM, Seagate, Varian) known as the National Storage Industry Consortium (NSIC). Our principal contact within NSIC has been HP. Together, HP and Los Alamos sponsored a highly successful 2-day workshop on CMR materials, held at LANL February 9-10, 1995. The workshop was attended by 10 companies, 12 universities, 5 national labs and representatives from NSIC and ARPA. We submitted two white papers to ARPA in collaboration with HP and several universities (Stanford, MIT, U. Minn). One of the white papers, concerning the development of a 100 Gbit $/$ in $^{2}$ segmented magnetic memory using MR and spin-polarized tunneling sensors in the magnetic read heads, was turned into a full $\$ 1.2 \mathrm{M} / \mathrm{yr}$. 5-year proposal at ARPA's request and submitted through NSIC. This was not funded, however. We also made presentations to DOE/AEP and ONR on similar subjects.

\section{Scientific Approach and Accomplishments}

We focused our efforts on learning to control the MR behavior of the manganite materials in thin film form, which is most suitable for device applications. This presents a particular challenge, because bulk materials are typically synthesized at high temperatures (1500-2000C) where the constituents are miscible. To create the kinds of thin-film multilayer structures, which we describe below, requires synthesis at much lower temperatures in order to prevent interlayer diffusion. This necessitated the investigation of the phase space for sample preparation: for example, the oxygen chemical potential (as determined by the oxygen partial pressure $\mathrm{PO}_{2}$ ) versus temperature. Accordingly, we mapped the film synthesis parameter space to determine the growth conditions necessary to achieve the following goals: (1) control of the effects of biaxial stress on the transport and 
magnetic properties of TMO films, both in single-layer films and in multilayers, and (2) development of multilayer structures of TMO films with other magnetic oxides to explore ways to tune the magnetoresistivity of these materials through the use of interlayer exchange coupling and spin-polarized tunneling.

Our approach combined the study of the basic physics of $(\mathrm{La}, \mathrm{Ca}) \mathrm{MnO}_{3}$ (primarily in bulk samples under a different LDRD project) with investigations of the effects of microstructure, chemical composition and geometry on thin-film device behavior (this project). We utilized our expertise in making thin-film TMO materials and multilayers, which has grown most recently from the Laboratory's high-temperature superconductivity initiative. We were able to characterize these films using a combination of $\mathrm{x}$-rays, electron microscopy, magnetization and (magneto) resistance.

Our standard characterization protocol for thin films was to first measure the lattice parameters (with x-rays), resistance and magnetization, and then - if interesting structural, transport or magnetic properties were found - to study the microstructure (TEM) and stoichiometry (Rutherford backscattering). This characterization protocol was developed to obtain fast sample turnaround.

We now summarize the physics issues inherent to $\mathrm{La}_{1-y} \mathrm{M}_{\mathrm{y}} \mathrm{MnO}_{3+x}$ materials. First, undoped $\mathrm{LaMnO}_{3}$ is an antiferromagnetic insulator below its transition temperature $\mathrm{T}_{\mathrm{N}} \approx$ $160 \mathrm{~K}$ [1]. The basic valence state of the ions is $\mathrm{La}^{3+}, \mathrm{Mn}^{3+}$ and $\mathrm{O}^{2-}$, so that as $\mathrm{M}=\mathrm{Ca}^{2+}$, $\mathrm{Ba}^{2+}$ or $\mathrm{Sr}^{2+}$ is added, charge conservation requires that a proportionate amount of $\mathrm{Mn}^{3+}$ be converted to $\mathrm{Mn}^{4+}$. When y exceeds $\approx 0.2$, conduction occurs via $\mathrm{d}$-spin electrons which hop between neighboring, spin-aligned $\mathrm{Mn}^{3+}$ and $\mathrm{Mn}^{4+}$ sites, producing ferromagnetism [2]. The maximum ferromagnetic $\mathrm{T}_{\mathrm{C}}$ occurs for $\mathrm{y} \approx 0.33$, where every $\mathrm{Mn}^{3+}$ statistically has a $\mathrm{Mn}^{4+}$ nearest neighbor. Furthermore, it is thought that the spins couple to one another to form spin clusters or polarons, accompanied by lattice distortions [3]. This spin-lattice structure is involved in the conduction process, giving rise to insulating behavior above $T_{c}$ and conducting behavior below $T_{C}$. A large negative $M R$ is observed very near $T_{C}$, because the applied field induces ferromagnetic correlations. Note that if $x \neq$ 0 the system compensates by producing cation vacancies or excess $\mathrm{Mn}^{4+}$ or both $(x>0)$. If $\mathrm{x}<0$, anion (oxygen) vacancies are introduced. These variations can affect the transport properties and $T_{c}$ greatly.

\section{Annealing Studies/ Oxygen Thermodynamics}

We compared two different deposition techniques, pulsed laser deposition (PLD) and rf magnetic sputtering, by synthesizing films $\sim 1000 \AA$ thick on $\mathrm{LaAlO}_{3}$ substrates. The 
films were deposited at deposition temperatures $T_{\mathrm{S}}$ between $500-900 \mathrm{C}$ and were characterized before and after annealing for 10 hours in oxygen at $950 \mathrm{C}$. We believe that the variation in conductivity and $T_{c}$ observed in many PLD films deposited at different temperatures is due to variations in $\mathrm{PO}_{2}$ during the deposition and annealing process. Consequently, we initiated a systematic study of the effects of deposition temperature, $\mathrm{PO}_{2}$ and annealing protocols on the magnetoresistivity of $\mathrm{La}_{1-y} \mathrm{Ca}_{y} \mathrm{MnO}_{3+x^{*}}$. Using thermogravimetric analysis on materials synthesized at different $\mathrm{PO}_{2}$ and $\mathrm{T}_{S}$, we developed the phase diagram shown in Fig. 1. Ideal films are made on the $\mathrm{x}=0$ line. Because of the need to work at the lowest possible deposition temperatures, we shifted our film growth method entirely to sputtering and planned to explore MBE growth in the future.

By varying the oxygen pressure and the substrate temperature during thin film growth, we have shown that the transport properties of unannealed lanthanum calcium manganite materials can vary by many orders of magnitude. Specifically, low oxygen pressures (below $10^{-7} \mathrm{~atm}$ ) and high temperatures $(>800 \mathrm{C}$ ) are required for increased conductivity in these materials. All of the unannealed films exhibit insulating transport properties. Annealing in a specially designed, electrochemically controlled, oxygen furnace with an in situ oxygen sensor indicated that very low oxygen pressures $\left(<10^{-8}\right.$ atm), and very high annealing temperatures $(900 \mathrm{C})$ are required to transform the films into materials that exhibit a distinct metal-to-insulator transition. Thus the ferromagnetic metallic state is probably metastable at temperatures below the annealing conditions. This low temperature metastability may explain the wide variation of physical properties observed by different research groups and for materials processed under differing synthetic conditions. We note that the metastable atomic arrangements are well quenched at room temperature, and no aging phenomena was observed in the films stored at room temperature. X-ray diffraction studies also indicated that an expansion of the c-lattice parameter occurs upon annealing. These effects are similar to what has been observed in the yttrium-barium-cuprate superconductors. 


\section{Deposition Studies on Lattice-Matched Substrates}

We studied the effects of lattice substrate epitaxy by depositing $(\mathrm{La}, \mathrm{Ca}) \mathrm{MnO}_{3}$ [LCMO] materials on a variety of lattice-matched isostructural substrates: lanthanum aluminate, strontium titanate, and neodymium gallate. These materials were selected to understand how controlled substrate-film epitaxial mismatch modifies the transport properties. Lanthanum aluminate has a smaller lattice parameter and thus induces a $1.6 \%$ compressive strain upon the manganite film. Neodymium gallate possesses a very similar lattice parameter to $(\mathrm{La}, \mathrm{Ca}) \mathrm{MnO}_{3}$, producing less than $0.1 \%$ mismatch. Growth on strontium titanate, however, induces a $1.3 \%$ tensile strain in the film layer. High resolution $x$-ray diffraction was used to characterize the lattice parameters of the films. Variations greater than $1 \%$ in the lattice parameter of the films deposited under identical conditions on different substrates were observed. This result indicates that large amounts of coherent interface strain can be produced during the thin-film growth process.

\section{Strain Layer Superlattices}

Transport experiments under hydrostatic pressure performed at Los Alamos [4] demonstrated a large pressure dependence to the resistivity in $\mathrm{La}_{67} \mathrm{Ca}_{33} \mathrm{MnO}_{3 \pm \mathrm{x}}$ materials. Relatively modest pressures $(<1.6 \mathrm{GPa})$ cause a decrease in the resistivity and an increase in $T_{c}$. This result suggested that we investigate the effects of epitaxial stress. Experimentally it is very difficult to apply a biaxial tensile/compressive stress to a brittle ceramic material. However, deformation has been successfully achieved in semiconductor systems by alternating thin film layers of the subject material with an epitaxial layer possessing a larger lattice parameter, forming a strain-layer superlattice.

We successfully adapted this semiconductor strain-layer approach and have fabricated multilayer superlattices by depositing alternating layers of $\mathrm{La}_{67} \mathrm{Ca}_{33} \mathrm{MnO}_{3 \pm x}$ with strontium titanate perovskites. These multilayer stacks exhibited multiple $\mathrm{X}$-ray superlattice satellite reflections, indicative of a highly ordered superlattice structure with a period of 290 $\AA ̊$ and d spacing corresponding to a highly strained material (see Figs. 2 and 3 ).

\section{Ferromagnetic Material Combinations}

The combination of soft ferromagnets with manganite materials may greatly reduce the field dependence of the magnetoresistivity by enhancing the effective field inside the manganite material. This can occur by two mechanisms: the first by a spin exchange mechanism and the second by local flux intensification using a high susceptibility ferromagnet. We began to deposit iron-zinc ferrite ferromagnetic materials in conjunction 
with the manganite films to explore these phenomena. The iron-zinc ferrites were chosen because they possess compatible thermodynamic stability with the manganites under the same deposition conditions, in addition to their desirable magnetic properties.

\section{Summary}

In conclusion, we accomplished the following:

- Established an excellent working relationship with several industrial organizations interested in MR materials, principally HP Corp. Together, HP and Los Alamos sponsored a highly successful 2-day workshop on MR materials, held at LANL February 9-10, 1995.

- Mapped out the $\mathrm{PO}_{2}$ - T phase diagram for producing metallic ( $\left.\mathrm{La}, \mathrm{M}\right) \mathrm{MnO}_{3}$ films. This enabled us to understand the variability of earlier reported studies and to make significant progress in learning to tailor the properties of TMO films.

- Demonstrated an ability to fabricate multilayer superlattices of alternating layers of LCMO material and $\mathrm{SrTiO}_{3}$. This is the first step in developing magnetic multilayers for use in possible TMO devices.

- Synthesized bulk polycrystalline samples for neutron scattering experiments designed to study structural instabilities in TMO materials.

- Published results in refereed journals and conference proceedings. 


\section{Publications}

1. M. F. Hundley, M. Hawley, R. H. Heffner, Q. X. Jia., J. J. Neumeier, J. Tesmer, J. D. Thompson and X. D. Wu, "Transport-Magnetism Correlations in the Ferromagnetic Oxide $\mathrm{La}_{0.7} \mathrm{Ca}_{0.3} \mathrm{MnO}_{3}$," Appl. Phys. Lett. 67, 860 (1995).

2. M. F. Hundley, J. J. Neumeier, R. H. Heffner, Q. X. Jia, X. D. Wu and J. D. Thompson, "Transport and Magnetism Correlations in Thin-Film Ferromagnetic Oxides," J. Appl. Phys. 79, 4535 (1996).

3. M. E. Hawley, X. D. Wu, P. N. Arendt, C. D. Adams, M. F. Hundley and R. H. Heffner, "Microstructural Study of CMR Films as a Function of Growth Temperature, AsDeposited and Annealed," Materials Res. Soc. 1996.

4. Despina Louca, W. Dmowski, T. Egami, E. L. Brosha, H. R. Roder and A. R. Bishop, "Evidence of Lattice Polarons in $\mathrm{La}_{1-\mathrm{x}} \mathrm{Sr}_{x} \mathrm{MnO}_{3}$ by Pulsed Neutron Diffraction," submitted to Phys. Rev. Letters (1996).

5. Despina Louca, E. L. Brosha and T. Egami, "Observation of Polaronic Lattice Distortion in $\mathrm{La}_{1-\mathrm{x}} \mathrm{Sr}_{\mathrm{x}} \mathrm{MnO}_{3}$ by Pulsed Neutron Scattering," submitted to Phys. Rev. Letters (1996).

6. M. E. Hawley, X. D. Wu, P. N. Arendt, C. D. Adams, M. F. Hundley, and R. H. Heffner, "Microstructural Study of CMR Films as a Function of Growth Temperature, as Deposited and Annealed," Mat. Res. Soc. Symp. Proceed., 401, 531 (1996).

7. M. E. Hawley, C. D. Adams, P. N. Arendt, E. L. Brosha, F. H. Garzon, R. J. Houlton, M. F. Hundley, R. H. Heffner, Q. X. Jia, J. Neumeier, and X. D. Wu, "CMR Films' Structure as a Function of Growth and Processing," J. Crystal Growth, submitted 1996. 


\section{References}

[1] P. Schiffer et al., "Low Temperature Magnetoresistance and the Magnetic Phase Diagram of $\mathrm{La}_{1-\mathrm{x}} \mathrm{Ca}_{\mathrm{x}} \mathrm{MnO}_{3}$," Phys. Rev. Lett. 75, 3336 (1995).

[2] C. Zener, Phys. Rev. 82, 403 (1951); P. W. Anderson and H. Hasegawa, Phys. Rev. 100, 675 (1955); P. G. deGennes, Phys. Rev 118, (1960) 141.

[3] D. Emin and N. L. H. Liu, Phys. Rev. B27, 4788 (1983); A. J. Millis, P. B. Littlewood and B. I. Shraiman, Phys. Rev. Lett. 74, 5144 (1995); J. Zang, A. R. Bishop and H. Röder, Phys. Rev B53, R8840 (1996); H. Röder, J. Zang and A. R. Bishop, Phys. Rev. Lett 76, 1356 (1996).

[4] J. J. Neumeier, et al., "Substantial Pressure Effects on the Electrical Resistivity and Ferromagnetic Transition Temperature of $\mathrm{La}_{1-x} \mathrm{Ca}_{x} \mathrm{MnO}_{3}$," Phys. Rev. B52 R7006 (1995). 


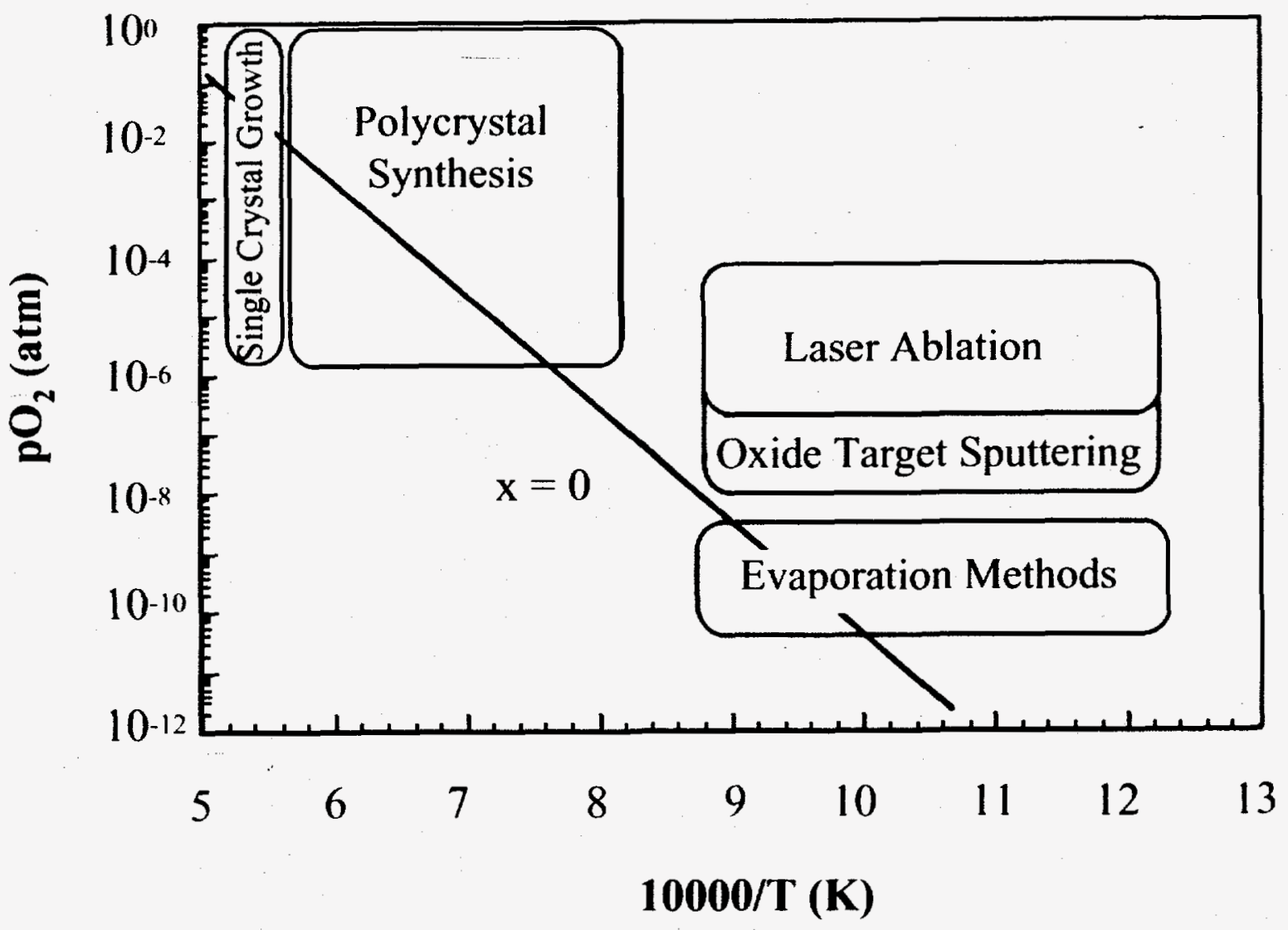

Figure 1. Approximate ranges of $\mathrm{La}_{0.67} \mathrm{Ca}_{0.33} \mathrm{MnO}_{3+\times}$ material synthesis techniques. The line of constant stoichiometric composition is represented by a sloped straight line. Above the $\mathrm{x}=0$ line excess oxygen is present (in the form of cation vacancies); below the line oxygen deficiencies exist (in the form of anion vacancies). 


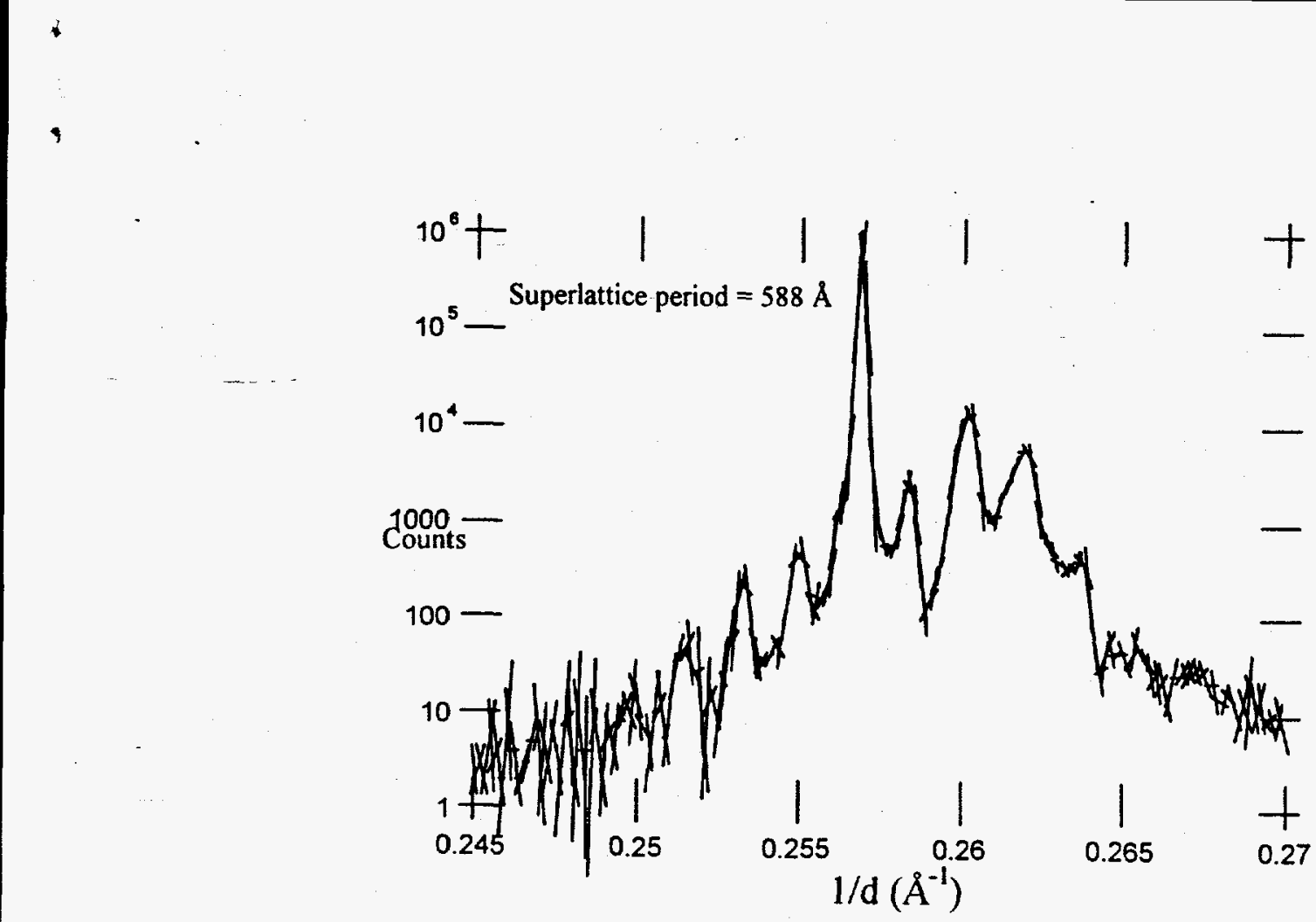

Figure 2. $\mathrm{La}_{0.67} \mathrm{Ca}_{0.33} \mathrm{MnO}_{3}-\mathrm{SrTiO}_{3}$ 10-layer strained superlattice consisting of $294 \AA$ A-thick layers of each material. The superlattice reflections indicate good structural periodicity.

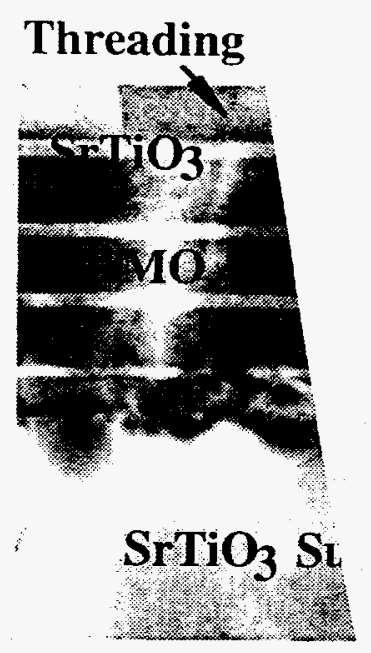

Figure 3. Bright-field TEM photomicrograph of $\mathrm{LCMO} / \mathrm{SrTiO}_{3}$ multilayer stack. The image shows excellent uniformity of the layer thickness, as well as threading dislocations that are spawned by the misfit dislocation network. 